\title{
Medienlinguistische Analyse der Kommunikation in Livestreams von Computerspielen
}

\author{
Medialinguistic analysis of communication \\ in livestream of computer games \\ Sławomir KOWALEWSKI ${ }^{1}$ \\ Uniwersytet Kazimierza Wielkiego w Bydgoszczy
}

\begin{abstract}
The main goal of this article is to linguistically analyse the livestream of computer games. The study has interdisciplinary nature - it is based mainly on media linguistics instruments. However, the paper also refers to certain aspects of text linguistics and, to a large degree, semiotics of multimodality. Livestreams are a relatively new media phenomenon and thus for linguists are unexplored and demand research. The following article will attempt to define as precisely as possible the concept of 'text' and 'medium' in a linguistic context. Furthermore, media information transfer during livestream will be analysed. Particular emphasis will be placed on to what extent the message and reception are supported by the phenomena of pluriand intermediality. The analysis is based on screenshots from the Internet platform Twitch.
\end{abstract}

Keywords: mediality, intermediality, text, livestream, computer game

\section{Zusammenfassung:}

Der vorliegende Beitrag setzt sich eine linguistisch fundierte Analyse der Livestreams von Computerspielen zum Ziel. Die Untersuchung hat einen interdisziplinären Charakter - sie basiert vor allem auf dem Instrumentarium der Medienlinguistik, bezieht sich jedoch auch auf einige Aspekte der Textlinguistik und, in hohem Maße, der Semiotik der Multimodalität. Livestreams sind eine relativ neue mediale Erscheinung und bilden aus diesem Grund eine Art

1 (D) https://orcid.org/0000-0002-1470-3984. 
unerforschten bzw. forschungsbedürftigen Raumes für die Medienlinguisten. Im vorliegenden Text wird der Versuch unternommen, die Begriffe ,Text' und ,Medium ' möglichst präzise im sprachwissenschaftlichen Kontext zu bestimmen und die mediale Übermittlung von Informationen während eines Livestreams zu analysieren. Ein besonderer Schwerpunkt wird darauf gelegt, inwiefern diese Übertragung und Rezeption durch die Phänomene der Pluriund Intermedialität unterstützt werden. Die Untersuchung basiert auf den Bildschirmfotos der Internetplattform Twitch.

Keywords: Medialität, Intermedialität, Text, Livestream, Computerspiel

\section{Einleitung}

Livestreams sind eine ziemlich neue mediale Erscheinung. Sie wecken gegenwärtig ein riesiges Interesse der Internetnutzer und ihre Popularität wächst immer weiter an. Bezüglich der Linguistik sind sie dennoch ein relativ unerforschter und somit forschungsbedürftiger kommunikativer Bereich.

Die Bedeutung der Bezeichnung ,Livestream‘ lässt sich der Wortbildung nach von ihren Bestandteilen ableiten, und zwar von den englischen Wörtern live, d. h. „in Echtzeit“, und stream, d. h. „Strom“ (in diesem Falle Datenstrom). Es ist damit die mittels Streaming - mittels eines Datenübertragungsverfahrens - übertragene Sendung gemeint, bei der die Daten bereits während der Übertragung angesehen oder angehört werden können, also in der Echtzeit und nicht danach (vgl. Internetquelle 1). Zu unterstreichen ist, dass der Begriff heutzutage immer mit dem Internet verbunden ist und die Liveübertragung von Bild und/oder Ton bedeutet. Es bestehen mehrere Portale, die solche Sendungen ermöglichen. $\mathrm{Zu}$ den bekanntesten gehören z. B. Youtube, Facebook oder Twitch.

Der vorliegende Beitrag setzt sich eine medienlinguistische Analyse der Livestreams von Computerspielen zum Ziel. Die Untersuchungen werden jedoch einen inter- bzw. transdisziplinären Charakter haben. Es wird nämlich auf das Instrumentarium der Textlinguistik Bezug genommen, um sich vor allem auf die sprachliche bzw. textuelle Ebene zu konzentrieren und die Abgrenzung zwischen Medienwissenschaft und Medienlinguistik deutlich zu machen. Darüber hinaus werden die Untersuchungen zum Teil auch die Aspekte der Game Studies und der Semiotik der Multimodalität verbinden. Durch die Analyse soll überprüft werden, auf welche Art und Weise und inwiefern die Livestreams von Computerspielen mehrere Modi und Codes bzw. unterschiedliche Medien miteinander verknüpfen. Die Untersuchung wird sich besonders auf die Textgestaltung bzw. Informationsübermittlung und den 
Kommunikationsverlauf beziehen sowie auf die Beständigkeit der multimodalen und -kodalen Verhältnisse bezüglich dieser Aspekte. Es muss darüber hinaus betont werden, dass der Beitrag ausschließlich die Livestreams auf der Streaming-Plattform Twitch anbetrifft, da sie unter den genannten Plattformen die meistgenutzte in der Gamingszene ist.

\section{Text und Medium - Begriffsklärung}

Versucht man den Terminus ,Text' zu bestimmen, muss man darauf Rücksicht nehmen, dass er wegen seiner Multidisziplinarität im höchsten Grade mehrdeutig ist. Es bestehen „Hunderte von Textdefinitionen“ (Heinemann, Heinemann 2002, S. 96). Darüber hinaus entstehen bei der Begriffsklärung sehr häufig Missverständnisse, unter anderem bzw. vor allem und paradoxerweise deswegen, weil er im Allgemeinen, d. h. im alltäglichen Gebrauch der Sprache präsent und verständlich ist. Aus diesem Grunde muss in Erwägung gezogen werden, dass die Bedeutung des Textbegriffs in Bezug auf die Textlinguistik völlig anders ist als in der Gemeinsprache oder in anderen Wissenschaften. Es sollte jedoch nie ausgeschlossen werden, dass sie mehr oder weniger transdisziplinär sein kann - die Sprache ist im Gebrauch nämlich äußerst selten bzw. sogar nie isoliert, d.h. sie bildet ein Gefüge von verschiedenen Zeichensystemen und kann von der Umgebung und der kommunikativen Situation nicht abgegrenzt werden (vgl. Holly 2009, S. 389). Die multimodale Rezeption eines Textes stellt also den Normalfall dar und soll aus diesem Grund nicht als eine Ausnahme gelten (vgl. Schneider, Stöckl 2011, S. 10). Durch die genannten Differenzen und Eigenschaften des Textuellen bzw. des Sprachlichen ist es höchst schwierig, eine eindeutige, unvoreingenommene Definition von ,Text ${ }^{`} \mathrm{zu}$ formulieren. Dennoch lassen sich bei der Begriffsstellung einige relevante Merkmale aus unterschiedlichen Textvorstellungen nennen, woraus folgende Interpretation abgeleitet werden kann: mit Text soll ein in sich durch sprachliche oder andere mediale Mittel kohäsives und kohärentes Gefüge verstanden werden, das unbedingt verbale Elemente beinhaltet, die jedoch nicht nur auf einer Modalität (Sinneswahrnehmung) basieren müssen. Text soll darüber hinaus als eine in gewissem Maße abgeschlossene Ganzheit mit mindestens einer kommunikativen Funktion betrachtet werden. In dem vorliegenden Beitrag wird also vorwiegend auf die Textvorstellung der multimodalen Textanalyse (vgl. Stöckl 2004) Bezug genommen, d. h. auf einen „übersprachlichen 
Textbegriff“" (Fix, Wellmann 2000, XIII), der an der Botschaftsübermittlung, am kommunikativen Handeln orientiert ist.

Ein weiterer Terminus, der unter den Wissenschaftlern sehr häufig unterschiedlich interpretiert wird, ist das Medium. Um jegliche Missverständnisse oder Mehrdeutigkeiten in der medialen Analyse zu vermeiden, müssen einige relevante Eigenschaften dieses Terminus präzisiert und erläutert werden. Dabei scheint es angebracht zu sein, zwei unterschiedliche Ansätze vorzubringen. Man muss am Anfang unterstreichen, dass sich die terminologische Unterscheidung zwischen ,Medium und ,Kommunikationsform' in der aktuellen deutschsprachigen Linguistik in gewissem Maße etabliert hat. In Bezug darauf kann hier also von einem engeren Medienbegriff gesprochen werden. Unter Medien werden demnach technische Medien verstanden, d. h. „konkrete materielle Hilfsmittel, mit denen Zeichen verstärkt, hergestellt, gespeichert und/oder übertragen werden können“ (Holly 1997, S. 69). Folgt man dieser Theorie, wären dann z. B. ein Fernseher, Computer oder andere digitale Träger unterschiedliche Medien, ebenso ein Buch und alle anderen analogen Phänomene. Sprache wäre hier dagegen, genauso wie sämtliche anderen Zeichensysteme (z. B. Ton und Bild), kein Medium.

Als zweites Medienkonzept kann das Grundprinzip der transkriptionstheoretischen Theorie erwähnt werden (vgl. Jäger 2004). In diesem Fall überlappen sich das Zeichen und das Medium teilweise semantisch. Das Medium wird also diesbezüglich im weiten Sinne betrachtet und gilt als Zeichenmedium. Daraus kann man schlussfolgern, dass es „prinzipiell keine nicht mediale, $d . h$. unvermittelte, nicht mediatisierte Kommunikation“ gibt. „Sprache und andere Zeichensysteme werden demnach auch als Medien gefasst. Individuelle und kulturell geteilte Bewusstseinsinhalte müssen immer erst entäußert, d. h. mediatisiert und enkodiert werden, um rezipiert werden zu können." (Schneider, Stöckl 2011, S. 16) Wenn man jedoch diese Auffassung näher betrachtet, gelangt man zu einigen strittigen Punkten. Schwierig wäre in dem Fall z. B. die eindeutige Abgrenzung zwischen ,Modus und ,Code' sowie zwischen ,Zeichenmedium' und ,Zeichensystem'. Eine Lösung wäre hier aber der Ansatz von Holly (2009), der die Begriffe ,Modalität‘ und ,Kodalität' unterscheidet:

Mit den Termini für die bisher erwähnten Erscheinungsformen von Sprache, ,Mündlichkeit' und ,Schriftlichkeit', fokussiert man die beiden Symbolsysteme nach den Herstellungsweisen, der materialen Qualität ihrer Zeichenoberflächen, also ,kodal': Lautsprache und Schriftsprache (als dritte wäre Gebärdensprache 
hinzuzunehmen). Mit dem Terminus ,Audiovisualität‘ wird dagegen auf ein bestimmtes Rezeptionspotenzial verwiesen, das mit den Sinnesorganen Ohr und Auge verbunden ist - also ,modal' (Holly 2009, S. 391).

Medium kann diesbezüglich als ein Oberbegriff für Kodalitäten und Modalitäten gelten und die beiden Kriterien verknüpfen. Demnach besteht aus mehreren Medien nur ein Gefüge, das mehrere Codes gebraucht oder über mehrere Modi übertragen wird (d.h. ein Gefüge, das multikodal oder multimodal ist).

Geht man diesen Unterscheidungen nach, kann man feststellen, dass der Begriff ,Medium' in Bezug auf Kommunikation nicht (nur) mehrdeutig, sondern eher mehrdimensional ist. Es ließen sich nämlich mehrere mediale Ebenen auflisten, die einander unterstützen. Dies kann grafisch folgendermaßen dargestellt werden (Abbildung 1):

\section{$\underline{\text { Medium als Institution }}$ \\ Medium als technischer/materieller Träger $\underline{\text { Medium als Zeichen (Modus und Code) }}$}

Abb. 1 Die allgemeine Darstellung der Medienhierarchie bezüglich der Kommunikation (eigene Bearbeitung)

Als ein grundlegendes Element der Kommunikation können die erwähnten Zeichensysteme und Modalitäten als Medien verstanden werden. Schrift und Bild gelten in diesem Falle als zwei unterschiedliche Medien in der Sphäre des Visuellen. Analog dazu sind auch die gesprochene Sprache und der nonverbale Ton zwei verschiedene auditive Medien. Darüber hinaus sind das Visuelle und das Auditive als zwei Medien zu verstehen. Weiter oben in der Hierarchie wird der Medienbegriff enger. Als Medien werden diesbezüglich technische bzw. materielle Träger verstanden, z. B. ein Computer oder ein Smartphone, die zu den Medien des digitalen Bereichs gehören, und z. B. ein Buch oder eine Zeitschrift, die sich außerhalb dieses Bereichs befinden, d. h. die analog sind. Diese materiellen Träger gelten als Medien, die die Übermittlung der grundlegenden semiotischen Medien (Modus und Code) möglich machen. Zum Schluss können hier noch die Medien als Institutionen hinzugefügt werden, z. B. Fernseh- oder Rundfunkkanäle, Verlage oder Internetplattformen wie Youtube oder Twitch (vgl. Schneider 
2017, S. 36). Zu unterscheiden wäre in diesem Kontext außerdem der Begriff ,Kommunikationsform'. Damit werden Texte oder eher Textsorten verstanden - sich wiederholende Gestaltungsvarianten bzw. Ordnungsmuster zwischen den Medien im weiteren Sinne, die mittels technischer Medien übertragen werden. Als Beispiele können hier Chat, Comic, Kochrezept oder Hypertext erwähnt werden.

\subsection{Pluri-, Multi-, Inter-, Trans-, Hypermedia}

Nach der Festlegung der Termini ,Text' und ,Medium' muss auf die Begriffe Multi- und Intermedia aufmerksam gemacht werden. Die Multimedia oder eher Plurimedia (vgl. Wasilewska-Chmura 2011, S. 33) - wie sie auch genannt werden können, um den mit Multimedia meistens falsch assoziierten obligatorischen Bezug auf die Sphäre des Digitalen abzulehnen - sind Gefüge, z. B. Texte, in denen mit verschiedenen Codes und über mehrere Sinnesmodalitäten (d. h. also mittels unterschiedlicher Medien) kommuniziert wird, z. B. verbal und nonverbal oder audiovisuell. Das Intermedium ähnelt in hohem Maße dem Begriff ,Plurimedium', mit dem Unterschied, dass innerhalb von intermedialen Gefügen die Zeichensysteme und Modi mehr oder weniger untrennbar sind und gleichzeitig rezipiert werden. Wenn man in diesem Falle auf die Rezeption eines einzelnen Mediums verzichten würde, wäre die übertragene Information unverständlich. In den plurimedialen Phänomenen können die Medien dagegen einzeln, selbständig funktionieren und nacheinander empfangen werden, ohne dass die Bedeutung der kommunikativen Botschaft verloren geht. In den Plurimedia sind die Kodalitäten und Modalitäten demzufolge nicht fest miteinander verbunden, ihre parallele Präsenz erleichtert jedoch die Rezeption (vgl. Clüver 1996, S. 26; Jensen 2008). Zu betonen ist hier aber, dass der Oberbegriff ,Medium' sowohl in Bezug auf Pluri- als auch auf Intermedia jeweils im weiteren Sinne verstanden wird.

Die Transmedialität wäre dagegen eine Art extrakompositionelle Erscheinung (vgl. Wolf 2002, S. 28), wobei betont werden muss, dass die Medien bezüglich dieses Phänomens eng verstanden werden sollten. Im Falle des Transmediums handelt es sich wiederum um Zusammenhänge zwischen mehreren Medien. Der Text innerhalb eines Mediums (eines technischen bzw. materiellen Trägers) wird aber in Bezug auf Transmedia in ein anderes Medium (einen anderen Träger) umgewandelt (vgl. Jenkins 2006, S. 96). Hier kann auch der Ausdruck transmedia intertextuality eingeführt werden (vgl. Kinder 1991), durch den man die Termini Transmedium 
und Intertext deutlich und explizit voneinander abgrenzen kann, wobei Intertexualität als Überschreitung der textuellen Grenzen vom narrativen Charakter betrachtet wird, das Transmedium sich dagegen auf die räumlichen bzw. medialen Grenzen bezieht.

In diesem Kontext können auch die Hypermedia erwähnt werden, d. h. die nichtlinearen und computervermittelten, durch Links und Knoten ermöglichten und automatisierten Verknüpfungen zwischen Medien im weiteren Sinne, z. B. innerhalb eines Textes (vgl. Storrer 2008, S. 318).

\section{Mediale Verknüpfungen und Kommunikation innerhalb eines Livestreams}

Im Folgenden sollen die Kommunikation und die Gestaltung medialer Verbindungen innerhalb eines Livestreams von Computerspielen auf der Internetplattform Twitch analysiert werden. Die Untersuchung basiert auf den unten stehenden Screenshots sowie auf ihren Auszügen (Abbildung 2 und 3):

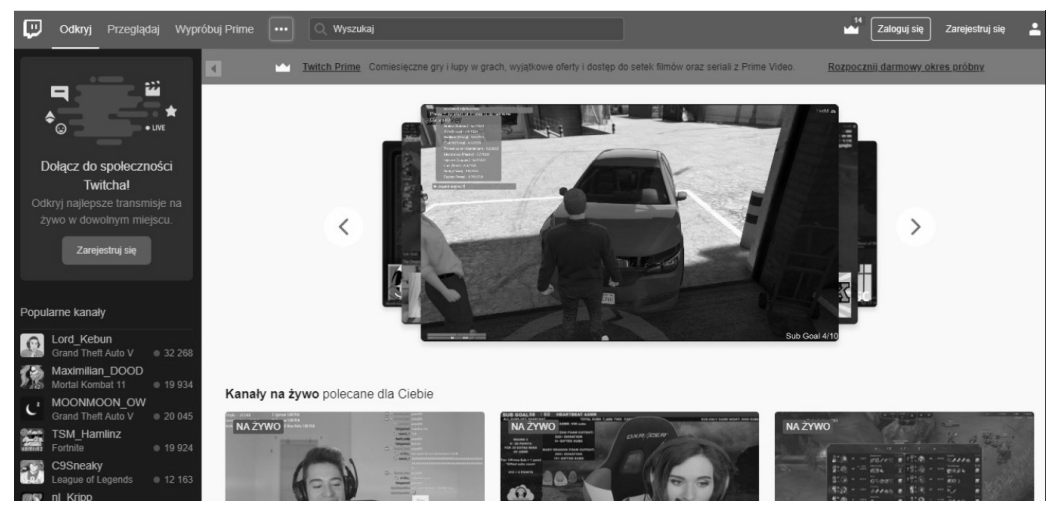

Abb. 2 Screenshot 1 - ein Beispielknoten aus der Internetplattform Twitch (eigene Bearbeitung) 


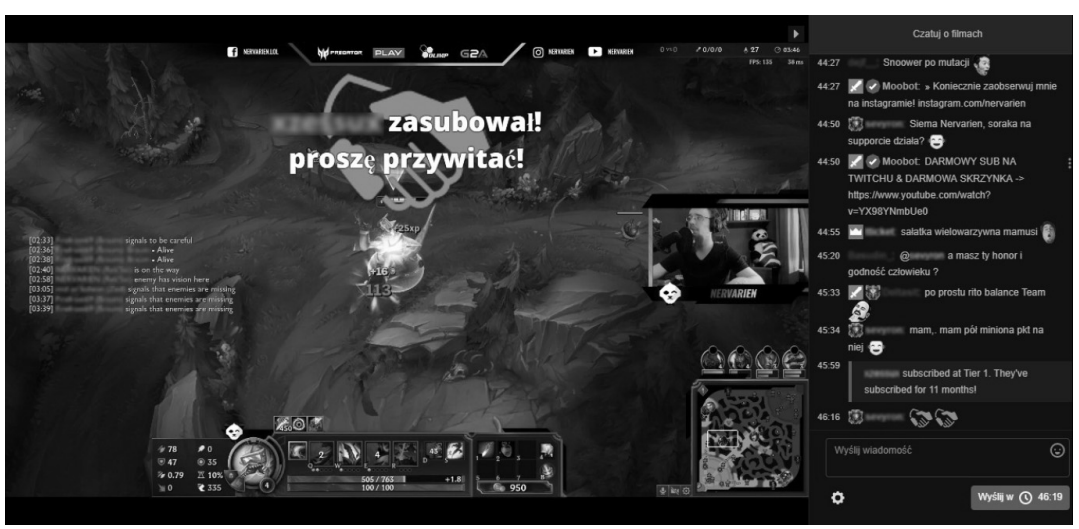

Abb. 3 Screenshot 2 - ein Beispielknoten des Livestreams von Computerspielen (eigene Bearbeitung)

Es lässt sich nicht verleugnen, dass die Internetplattform Twitch einen hypermedialen bzw. hypertextuellen Charakter aufweist (Screenshot 1). Die einzelnen Elemente werden in Knoten eingeteilt, die weiter mit Links verbunden sind, was ihre globale Kohäsion und Kohärenz ermöglicht. Die Links werden durch das Anklicken verschiedener Schaltflächen aktiviert. Der Knoten im Ganzen ähnelt strukturell anderen Internetseiten des World Wide Web.

Die Schaltflächen sind sehr häufig monokodale und monomodale, d. h. monomediale Gefüge. Viele von ihnen bestehen nämlich ausschließlich aus verbalen visuellen Elementen (Abbildung 4).

\section{Odkryj Przeglądaj Wypróbuj Prime}

Abb. 4 Auszüge aus dem Screenshot 1 - verbale Schaltflächen.

Links sind Registerkarten zu sehen, rechts befinden sich die Hyperlinks zur Registrierung oder Anmeldung (eigene Bearbeitung).

Andere Schaltflächen gelten dagegen als lediglich nonverbale visuelle Zeichen (Abbildung 5).

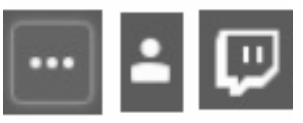

Abb. 5 Auszüge aus dem Screenshot 1 - nonverbale Schaltflächen. Von links: DropDown-Menü, Profil- oder Kontoeinstellungen, Startseite (eigene Bearbeitung) 
Es bestehen jedoch auch unterschiedliche multikodale und monomodale (in diesem Falle visuelle) Mischformen. Die Beständigkeit der Verbindungen zwischen den Zeichen verschiedener Kodalitäten (d. h. zwischen Medien im weiteren Sinne) ist hier aber nicht so stark ausgeprägt. Sie existieren eher parallel nebeneinander und unterstützen semantisch einander, sind deswegen als Plurimedia zu betrachten (Abbildung 6).

\section{Wyszukaj}

Lord_Kebun

Grand Theft Auto V 32268

Abb. 6 Auszüge aus dem Screenshot 1 - multikodale Schaltflächen. Links oben sieht man eine eingebaute Suchmaschine, rechts oben den Link zu Informationen über die Twitch-Prime-Mitgliedschaft. Unten befindet sich ein Link zu einem konkreten Livestream bzw. Kanal (eigene Bearbeitung).

Als das einzige multikodale und multimodale Element gilt hier die Videowahl in der Mitte des Knotens (Abbildung 7).

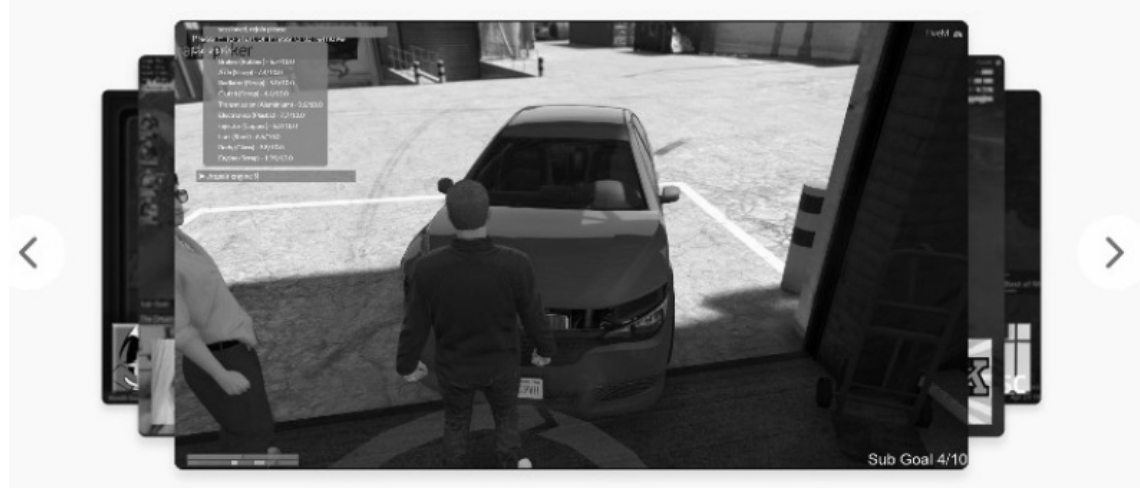

Abb. 7 Auszug aus dem Screenshot 1 - eine Gruppe von Schaltflächen mit Links zu bestimmten Kanälen (eigene Bearbeitung)

Dieses Element scheint intermedial zu sein, es handelt sich hier nämlich um die Audiovisualität, d. h. um Zusammenhänge zwischen den visuellen und akustischen Zeichen, die gleichzeitig rezipiert werden und relativ fest miteinander verbunden sind. Wenn man aber berücksichtigt, dass diese Videos eher nur als eine Schaltfläche betrachtet werden, als ein Hyperlink, der zum 
Knoten mit einem konkreten Livestream führt, kann man zu dem Schluss kommen, dass die Bedeutung des Auditiven irrelevant ist. Wenn der Rezipient das Video stummschaltet, bleibt die Bedeutung des Hyperlinks relativ unverändert.

Die Knoten eines Livestreams (Screenshot 2) sind in Bezug auf das Mediale interessanter. Jeder dieser Knoten besteht nämlich aus einem bewegten Bild und dem Audio, die live übertragen werden, mit denen auch in jedem Fall ein Chatfenster einhergeht. Äußerst interessant ist in Bezug darauf (d. h. innerhalb dieses Knotens) der Aspekt der Kommunikation bzw. der Informationsübertragung während eines Streams, denn in jedem Stream ist die Kommunikation mehrdimensional, was am Beispiel folgender Grafik dargestellt werden kann (Abbildung 8, vgl. Kirschner 2013, S. 166):

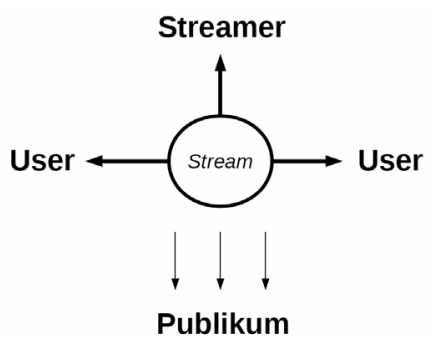

Abb. 8 Grafische Darstellung der Kommunikation in einem Stream (vgl. Kirschner 2013, 166)

$\mathrm{Zu}$ betonen ist, dass hier unter Publikum nicht alle Beobachter verstanden werden, sondern lediglich die nicht angemeldeten Zuschauer, d. h. alle Rezipienten, die keinen Twitch-Account haben oder diejenigen, die sich nicht eingeloggt haben. Die User sollen dagegen als die angemeldeten Betrachter verstanden werden. Das Publikum rezipiert das, was innerhalb des Livestreams vom Streamer (bzw. im Stream selbst) und von den Usern produziert wird, hat aber keine Möglichkeit, sich zu äußern. Die User können dagegen beiderseitig kommunizieren, mit dem Streamer sowie miteinander.

Noch interessanter scheint die Kommunikation in Bezug auf die Livestreams von Computerspielen zu sein, dort lassen sich nämlich noch weitere Ebenen der Kommunikation erwähnen (Abbildung 9): 


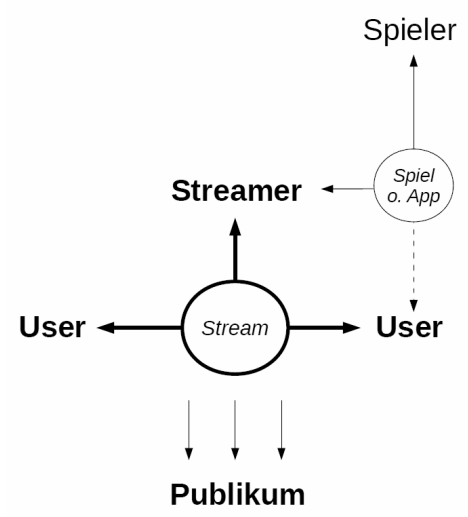

Abb. 9 Grafische Darstellung der Kommunikation in einem Stream von Computerspielen (eigene Bearbeitung)

Im Falle der Livestreams von Computerspielen kommuniziert der Streamer, der hier auch als einer der Spieler zu betrachten ist, mit anderen Spielern. Diese Informationsübertragung ist beiderseitig und erfolgt entweder über die in das Spiel eingebauten Kommunikatoren, wie z. B. der Ingame-Chat, oder über externe Kommunikationsapplikationen, z. B. Skype, Teamspeak oder Discord. In einigen Fällen ist auch die Kommunikation zwischen den Usern und Spielern möglich, wenn z. B. ein User einer der Spielenden ist.

Im Folgenden werden die bestimmten Kommunikationsebenen und/oder Kommunikationsrichtungen in Bezug auf ihre medialen Eigenschaften analysiert, d. h. bezüglich ihrer Modalität und Kodalität sowie der Beständigkeit der Zusammenhänge zwischen Medien.

\section{1) Produzent: Streamer; Rezipient: User, Publikum}

a. Webcam und die gesprochene Sprache des Streamers

Die Kommunikation über die Webcam erfolgt mittels visueller und nonverbaler Medien. Es handelt sich in diesem Falle um ein bewegtes nonverbales Bild, auf dem die reale Umgebung des Streamers und der Streamer selbst angezeigt werden. Hier sind in Bezug auf die Kommunikation die Mimik und Gestik von großer Bedeutung. Andererseits ist jedoch wichtig zu betonen, dass diese Informationsübertragung mit der auditiven (sowohl verbalen als auch nonverbalen) Ebene äußerst stark verbunden ist, d.h. mit der gesprochenen Sprache des Streamers und allen anderen Geräuschen seinerseits sowie aus der Umgebung, die technisch gesehen durch das Mikrofon übertragen werden. Man kann sogar behaupten, dass einige Bestandteile 
der Informationsvermittlung verloren gehen, wenn man auf die Rezeption eines der Modi verzichtet. Aus diesem Grund kann man schlussfolgern, das die Verbindungen zwischen den Medien im weiteren Sinne in diesem Falle als Intermedia zu betrachten sind.

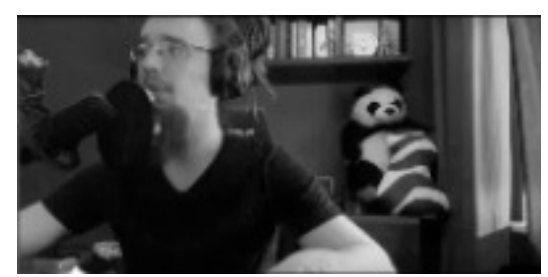

Abb. 10 Auszug aus dem Screenshot 2 - Bild aus der Webcam (eigene Bearbeitung)

\section{b. Stream-Overlay}

Mit Stream-Overlay sind alle grafischen Elemente zu verstehen, die sich oberhalb des übertragenen Bildes befinden und dieses ergänzen bzw. schmücken. Vor allem handelt es sich hier um eine Art visueller Gestaltung des Streamdesigns. Das Overlay trägt jedoch nicht selten sehr viel zur Kommunikation bei, indem es viele Informationen über den Streamer und das Livestream beinhaltet (z. B. über den Nicknamen des Streamers oder die Sponsoren, Fanpages, usw.) sowie das Head-up-Display ermöglicht. Das Overlay ist also in hohem Maße visuell und nonverbal gestaltet (vor allem Standbild, seltener Animationen), kann jedoch manchmal ein plurimediales Gefüge mit verbalen visuellen Elementen gestalten.

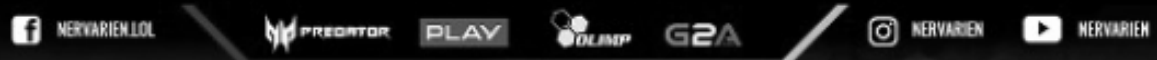 - $\times$ MERUARIEM}

Abb. 11 Auszüge aus dem Screenshot 2 - multikodale Elemente des Stream-Overlays (eigene Bearbeitung)

\section{c. Benachrichtigungen}

Mit Benachrichtigungen werden jegliche Informationen über Geschehnisse während des Streams verstanden, z. B. wenn ein User einen Kanal abonniert oder den Streamer dotiert. Die Benachrichtigungen werden vor allem visuell verbal angezeigt - als ein Text im bzw. über dem Video oder als eine automatische Nachricht im Chat. Mit diesen gehen auch visuelle nonverbale 
Elemente einher, z. B. ein Standbild oder eine Animation im Video, sowie auditive nonverbale Komponenten, z. B. Töne oder Musik. Standbilder, Animationen, Töne oder Musik bekommen, obwohl sie in hohem Grade nonverbal sind, meistens eine explizite und eindeutige semantische Bedeutung, indem jeder konkreten Benachrichtigung ein konkretes Bild, ein konkreter Ton etc. zugeschrieben wird. Bei den Benachrichtigungen werden manchmal auch auditive verbale Elemente wiedergegeben, z. B. die Sprache in der Musik oder ein durch Sprachsynthese generierter Text ${ }^{2}$. Die genannten Modi und Codes existieren in Bezug auf die Benachrichtigungen eher parallel nebeneinander, sind mehr oder weniger voneinander unabhängig und ergänzen die Botschaft. Man spricht hier also von Plurimedia.

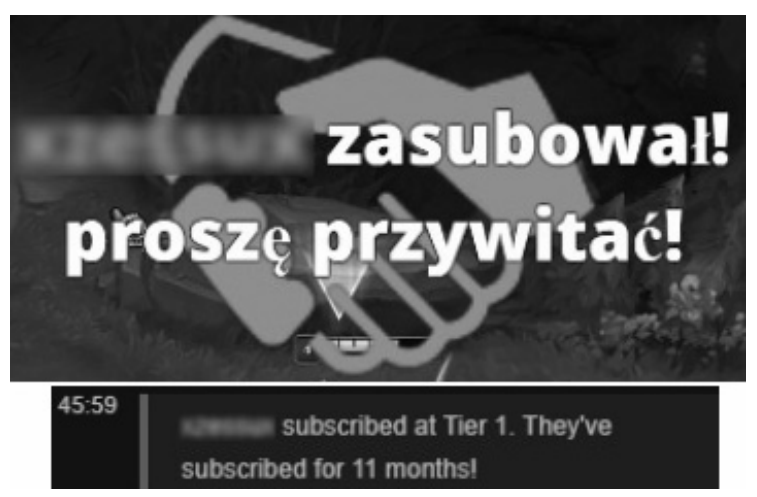

Abb. 12 Auszüge aus dem Screenshot 2 - oben: die Benachrichtigung über dem Video, unten: die Benachrichtigung im Chat (eigene Bearbeitung)

\section{d. Chat-Nachrichten}

Der Streamer hat auch die Möglichkeit, sich visuell und verbal im Chat zu äußern, die aber relativ selten genutzt wird, da die auditive verbale Kommunikation über Mikrofon präzise genug und zeitökonomisch ist. Die meisten Chat-Nachrichten des Streamers werden automatisch generiert (z. B. bei den Benachrichtigungen). Zu betonen ist hier darüber hinaus, dass die Kommunikation im Chat auch nonverbale visuelle Elemente beinhaltet, z. B. die Emoticons. Diese sind aber mit den verbalen Komponenten nicht fest verknüpft, man kann also in diesem Falle von Plurimedia sprechen.

${ }^{2}$ Dieser Text wird jedoch seitens User produziert (vgl. Punkt 2b). 
e. Bild und Ton des Spiels

Obwohl der Spielinhalt im Grunde genommen einen anderen Produzenten hat (die Spielautoren), kann man in Bezug auf Livestreams annehmen, dass hier die Informationen vom Streamer produziert werden, da er sie zum Zwecke des Streamings gewählt hat. Unter dieser Voraussetzung wird der Streamer zu dem sekundären und zugleich relevanteren Produzenten. Die Elemente des Spiels können (je nach Spiel) verbal und nonverbal sowie visuell und akustisch wiedergegeben werden. Einige von ihnen sind den Plurimedia zuzuschreiben, die anderen dagegen sind intermedialen Verhältnissen untergeordnet.
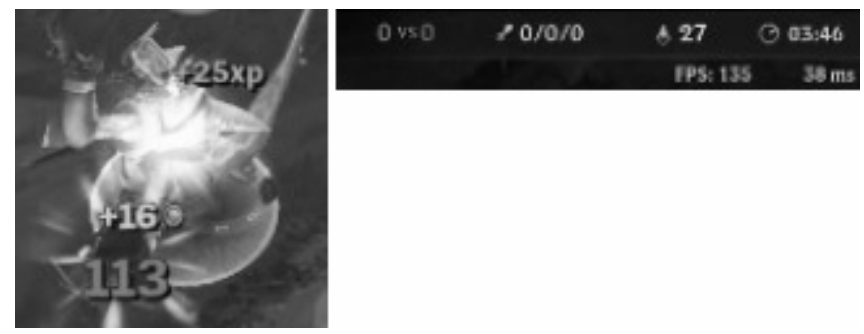

Abb. 13 Auszüge aus dem Screenshot 2 - Elemente des übertragenen Spiels. Links: intermediales Gefüge von visuellen und auditiven verbalen und nonverbalen Elementen, rechts: plurimediale visuelle sowie verbale und nonverbale Elemente (eigene Bearbeitung)

\section{2) Produzent: User; Rezipient: Streamer, User}

\section{a. Nachrichten im Chat}

Die Möglichkeiten des Kommunizierens seitens User sind in hohem Maße beschränkt. Die User können sich nämlich lediglich per Chat äußern. Diese Kommunikation ist monomodal - basiert nur auf visuellen Sinnesmodalitäten. Es gibt keine technische Möglichkeit, Audioaufnahmen zu senden. Die Informationsübermittlung im Chat ist dennoch multikodal, da neben der verbalen Elemente auch Emoticons verschickt werden können. Diese haben auf der Plattform Twitch sehr häufig eine konkrete semantische Bedeutung; das sog. Kappa-Emoji weist z. B. auf Ironie oder sogar auf Sarkasmus in der Äußerung hin. Die Emoticons sind, wie erwähnt, mit den verbalen Zeichensystemen relativ schwach verknüpft, sie bilden aus diesem Grunde Plurimedia und erweitern nur die Semantik des Sprachlichen sowie drücken das Emotionale aus. 


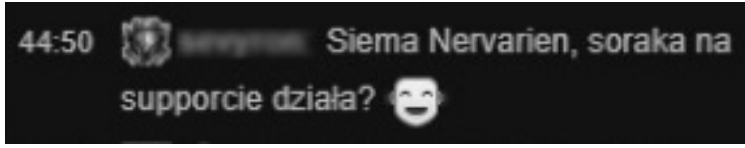

Abb. 14 Links: Auszug aus dem Screenshot 2 - eine plurimediale Beispielnachricht im Chat, rechts: das Kappa-Emoji (eigene Bearbeitung)

\section{b. Nachricht bei einer Benachrichtigung}

Es handelt sich hier um die im Punkt 1c erwähnte Nachricht eines Users, die bei einer Benachrichtigung mittels Sprachsynthese auditiv verbal wiedergegeben (und manchmal auch visuell verbal angezeigt) wird. $\mathrm{Zu}$ betonen ist hier, dass diese Nachrichten ausschließlich nach dem Dotieren des jeweiligen Kanals abgespielt werden können und deswegen seltener als diejenigen im Chat genutzt werden. Obwohl diese Informationsübertragung nur in Bezug auf plurimediale (multikodale und multimodale) Benachrichtigungen möglich ist, ist sie als monomedial (auditiv verbal) zu betrachten, da die Bedeutung der Nachricht nicht mit der Bedeutung der Benachrichtigung selbst verbunden ist - sie ist also als ein separates kommunikatives Element zu verstehen. Seltener - wenn die visuelle verbale Ebene der Nachricht besteht und angezeigt wird - wird die Botschaft den Plurimedia zugeschrieben.

\section{3) Produzent: Streamer; Rezipient: Spieler (oder umgekehrt)}

Diese kommunikative Ebene ist weniger relevant, da sie sich vor allem auf das Spiel bzw. die Spieler und weniger auf die Rezipienten eines Livestreams bezieht. Zu unterstreichen ist auch, dass sich ihre Möglichkeiten je nach Spiel unterscheiden können. Trotzdem ist es angebracht, einige Aspekte dieser Kommunikation zu erwähnen, da sie teilweise auch live übertragen werden.

a. Ingame-Chat

Jedes Online-Spiel verfügt prinzipiell über einen Ingame-Chat. Diese Form der Kommunikation ähnelt in hohem Grade einem Chat im WWW, mit dem Unterschied, dass innerhalb eines Spiels in den meisten Fällen auf Emoticons verzichtet wird ${ }^{3}$. Die Kommunikation in einem Ingame-Chat ist also monomedial (verbal visuell).

b. andere in das Spiel eingebauten kommunikativen Möglichkeiten $\mathrm{Zu}$ dieser Kategorie gehören die artverschiedensten Formen, z. B. der auditive verbale (monomediale) Voice-Chat, die nonverbalen visuellen und auditiven

\footnotetext{
${ }^{3}$ Abgesehen von den Emoticons, die mittels Interpunktionszeichen gebildet werden.
} 
Zeichen (mit denen manchmal verbale visuelle und automatisch generierte Chat-Nachrichten einhergehen), die beispielsweise mit Mausgesten aktiviert und auf dem Bild des Spiels angezeigt werden. All diese Formen sollen die Kommunikation beschleunigen und präzisieren. Sie sind als Intermedia zu betrachten, da hier die Modalitäten und Kodalitäten eng verbunden sind und relativ gleichzeitig rezipiert werden.
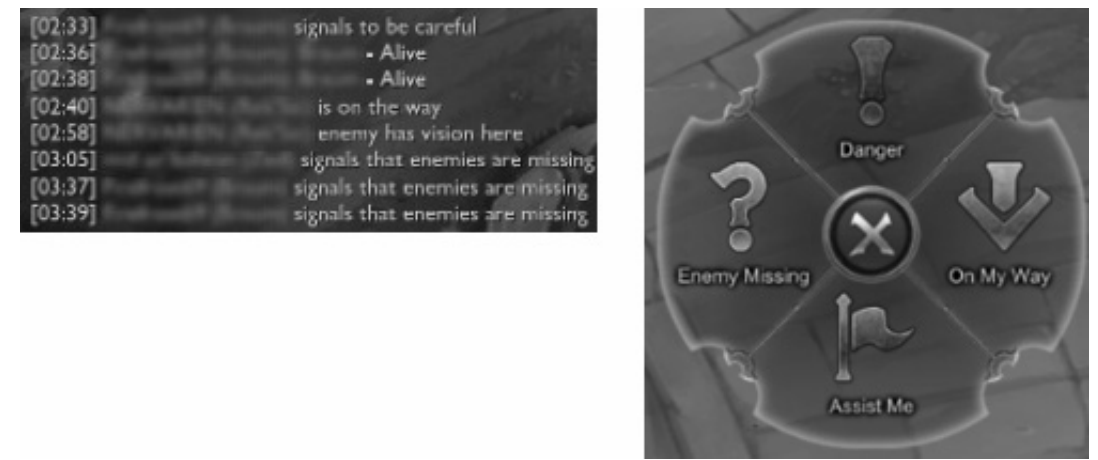

Abb. 15 Auszüge aus dem Spiel „League of Legends“ - links: die automatisch generierten verbalen visuellen Chat-Nachrichten, rechts: die Mausgesten mit sog. Pings, d. h. einer Art Kurzbefehlen (eigene Bearbeitung)

c. Kommunikation über eine externe Applikation

Dies betrifft vor allem externe Voice-Kommunikatoren, die verbale auditive, d. h. monomediale Informationsübertragungen ermöglichen. Einige von ihnen bieten jedoch auch die Möglichkeit, Videos zu übertragen. Dadurch wird die Kommunikation um visuelle nonverbale Elemente bereichert, wodurch eine intermediale Kommunikation entstehen kann. Dies wird aber während des Spiels nicht gebraucht, weil der Spielende meistens auf das Bild des Spiels konzentriert bleibt. Als die bekanntesten Beispiele solcher externen Programme gelten Skype, Discord oder Teamspeak.

\section{Fazit}

Aus der Analyse lässt sich schlussfolgern, dass die Internetplattform Twitch aus textuellen sowie nicht-textuellen Elementen besteht. Ihre Struktur weist die Eigenschaften eines Hypermediums bzw. Hypertextes auf, die Plattform besteht nämlich aus nichtlinear geordneten und rezipierten Knoten und Hyperlinks, die ihre globale Kohäsion und Kohärenz ermöglichen, sodass die Plattform teilweise den anderen Seiten des World Wide Web ähnelt. 
Die Schaltflächen können mittels verschiedener Kodalitäten gestaltet und durch unterschiedliche Sinnesmodalitäten rezipiert werden. Einige von ihnen sind monokodal und -modal, d.h. also monomedial. Die anderen dagegen können als Plurimedia betrachtet werden. Die Schaltfläche mit dem Video (die Wahl der Livestreams) kann unter Umständen auch als Intermedium betrachtet werden, fokussiert man aber seine Funktion (Gestaltung des Hypermedialen, der globalen Kohärenz und Kohäsion), ist sie als Plurimedium zu verstehen.

Der Knoten eines konkreten Livestreams der Computerspiele besteht jeweils aus dem live übertragenen Video und dem Chat. Die Kommunikation während solch eines Livestreams ist mehrdimensional. Sie besteht nämlich aus drei Hauptebenen. Als die erste Ebene gilt hier die Kommunikation zwischen dem Streamer und dem Publikum sowie den Usern, wobei der Streamer der Produzent ist. In der zweiten Ebene sind die User Produzenten, der Streamer ist dagegen als Rezipient zu betrachten. Die dritte Ebene, die nur für Livestreams von Computerspielen charakteristisch ist, ist die beiderseitige Informationsübertragung zwischen dem Streamer und den Spielern. Jede genannte Dimension der Kommunikation während eines solchen Livestreams weist bestimmte, typisch mediale Charakteristika auf. Diese lassen sich am einfachsten anhand folgender Tabelle zusammenfassen (Tabelle 1):

Die Informationsübertragung über die Webcam und das Mikrofon ist einerseits medial visuell und nonverbal (eventuell paraverbal). Die gesprochene Sprache des Streamenden ist als ein verbales auditives Medium zu betrachten. Das daraus bestehende Gefüge ist sowohl multikodal als auch multimodal und bildet ein Intermedium. Das Stream-Overlay besteht manchmal aus lediglich visuellen nonverbalen Elementen und kann deswegen als monomedial verstanden werden. In den meisten Fällen kommt aber noch das visuelle Verbale hinzu. Es entsteht dann ein multikodales monomodales Gefüge, das zu den Plurimedia gezählt werden kann. Die Benachrichtigungen bestehen immer aus verbalen visuellen und auditiven Medien sowie aus dem verbalen und nonverbalen Audio, sind also multikodal und multimodal. Die Medien sind hier aber eher unabhängig und können deswegen als Plurimedia verstanden werden. Die Chat-Nachrichten, die sowohl von dem Streamer als auch von den Usern produziert werden, sind einerseits immer verbal visuell, können aber andererseits durch Emoticons, d. h. durch das visuelle nonverbale Medium bereichert werden. Ohne Emojis sind sie monomedial, mit ihnen dagegen multikodal und plurimedial. Jedes Spiel besteht jeweils aus dem nonverbalen Visuellen. Es muss aber auch über visuelle verbale Elemente verfügen. 
Das Auditive ist nicht obligatorisch, aber in den meisten Fällen präsent. Die Elemente eines Spiels sind also multikodal und können auch multimodal sein. Sie können sowohl pluri- als auch intermediale Zusammenhänge bilden. Die Nachrichten bei einer Benachrichtigung sind immer verbal auditiv und werden mit Hilfe der Sprachsynthese wiedergegeben. Mitunter können sie auch mit dem visuellen Verbalen ergänzt werden. Sie sind also entweder monomedial oder multimodal plurimedial. Der Ingame-Chat ist im Prinzip ausschließlich verbal und visuell, also monomedial. Andere in das Spiel eingebaute Kommunikationsmöglichkeiten können sehr unterschiedlich sein und sowohl verbal als auch nonverbal sowie auditiv und visuell gestaltet werden. Sie können darüber hinaus monomedial, plurimedial oder intermedial sein, alle Varianten sind hier demnach prinzipiell möglich. Die Kommunikation über eine externe Kommunikationsapplikation ist verbal und auditiv (monomedial), kann aber auch nonverbal visuell ergänzt werden, wobei dann ein multikodales und -modales intermediales Gefüge entsteht.

Tbl. 1 Zusammenfassung der Analyse von medialen Verhältnissen in der Kommunikation während eines Livestreams von Computerspielen (eigene Bearbeitung)

\begin{tabular}{|c|c|c|c|c|c|c|c|c|c|c|}
\hline \multirow{3}{*}{\multicolumn{2}{|c|}{$\begin{array}{c}\text { Kommunikative Ebene } \\
\text { eines Livestreams }\end{array}$}} & \multicolumn{4}{|c|}{$\begin{array}{c}\text { Mediale Qualitäten der } \\
\text { gebrauchten } \\
\text { Zeichensysteme } \\
\end{array}$} & \multirow{2}{*}{\multicolumn{3}{|c|}{$\begin{array}{l}\text { Präsenz } \\
\text { mehrerer } \\
\text { Medien }\end{array}$}} & \multirow{2}{*}{\multicolumn{2}{|c|}{$\begin{array}{l}\text { Beständigkeit der } \\
\text { medialen } \\
\text { Zusammenhänge }\end{array}$}} \\
\hline & & \multicolumn{2}{|c|}{ Code } & \multicolumn{2}{|c|}{ Modus } & & & & & \\
\hline & & 宽 & 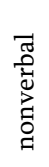 & 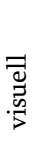 & :己 & 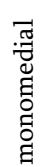 & 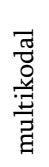 & 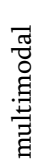 & 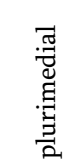 & 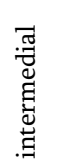 \\
\hline \multirow{5}{*}{ 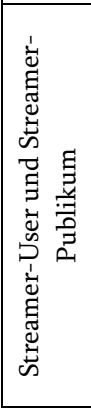 } & $\begin{array}{c}\text { a - Webcam und die } \\
\text { gesprochene Sprache } \\
\text { des Streamers }\end{array}$ & + & + & + & + & - & + & + & - & + \\
\hline & b-Stream-Overlay & $+/-$ & + & + & - & $+/-$ & $+/-$ & - & $+/-$ & - \\
\hline & $\begin{array}{c}\mathrm{c}- \\
\text { Benachrichtigungen }\end{array}$ & + & + & + & + & - & + & + & + & - \\
\hline & d-Chat-Nachrichten & + & $+/-$ & + & - & $+/-$ & $+/-$ & - & $+/-$ & - \\
\hline & $\begin{array}{c}\text { e - Bild und Ton des } \\
\text { Spiels }\end{array}$ & + & + & + & $+/-$ & - & + & $+/-$ & $+/-$ & $+/-$ \\
\hline 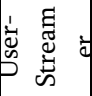 & $\begin{array}{c}\text { a - Nachrichten im } \\
\text { Chat }\end{array}$ & + & $+/-$ & + & - & $+/-$ & $+/-$ & - & $+/-$ & - \\
\hline
\end{tabular}




\begin{tabular}{|c|c|c|c|c|c|c|c|c|c|c|}
\hline & $\begin{array}{c}\mathrm{b}-\text { Nachricht bei } \\
\text { einer } \\
\text { Benachrichtigung }\end{array}$ & + & - & $+/-$ & + & $+/-$ & - & $+/-$ & $+/-$ & - \\
\hline \multirow{3}{*}{ 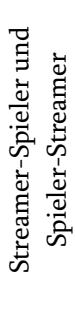 } & a-Ingame-Chat & + & - & + & - & + & - & - & - & - \\
\hline & $\begin{array}{l}\mathrm{b}-\text { andere in das } \\
\text { Spiel eingebauten } \\
\text { kommunikativen } \\
\text { Möglichkeiten }\end{array}$ & \multicolumn{9}{|c|}{$\begin{array}{l}\text { Je nach Spiel können sie in Bezug auf die Medialität } \\
\text { unterschiedlich gestaltet werden. }\end{array}$} \\
\hline & $\begin{array}{c}\text { c- Kommunikation } \\
\text { über eine externe } \\
\text { Applikation }\end{array}$ & + & $+/-$ & $+/-$ & + & $+/-$ & $+/-$ & $+/-$ & - & $+/-$ \\
\hline
\end{tabular}

Medial gesehen ist also die Kommunikation während der Livestreams von Computerspielen sehr komplex und heterogen. In den meisten Fällen besteht sie aus Elementen, bei denen mehrere Medien präsent sind, d. h. Plurimedia oder Intermedia. Relativ oft verläuft die Kommunikation aber auch monomedial.

\section{Literaturverzeichnis}

Clüver, C. (1996). Intermediality and Interart Studies. In: Arvidson, J.; Askander, M.; Bruhn, J.; Führer, H. (Hrsg.): Changing Borders. Contemporary Positions in Intermediality. (S. 19-37). Lund: Intermedia Studies Press.

Fix, U., Wellmann, H. (2000). Sprachtexte - Bildtexte. Bemerkungen zum Symposion "Bild im Text - Text und Bild" vom 6.-8. April 2000 in Leipzig. In: Fix, U.; Wellmann, H. (Hrsg.): Bild im Text, Text im Bild. Heidelberg: Winter, XI-XVII.

Heinemann, M., Heinemann, W. (2002). Grundlagen der Textlinguistik. Interaktion Text - Diskurs. Tübingen: Niemeyer.

Holly, W. (1997). Zur Rolle von Sprache in Medien. Muttersprache 107, 1, 64-75.

Holly, W. (2009). Der Wort-Bild-Reißverschluss. Über die performative Dynamik der audiovisuellen Transkriptivität. In: Linke, A.; Feilke, H. (Hrsg.): Oberfläche und Performanz. Untersuchungen zur Sprache als dynamischer Gestalt. (S. 389-406). Tübingen: Niemeyer.

Jäger, L. (2004). Wieviel Sprache braucht der Geist? Mediale Konstitutionsbedingungen des Mentalen. In: Jäger, L., Linz, E. (Hrsg.): Medialität und Mentalität. Theoretische und empirische Studien zum Verhältnis von Sprache, Subjektivität und Kognition. (S. 15-42). München: Fink.

Jenkins, H. (2006). Convergence Culture: Where Old and New Media Collide. New York, London: NYU Press.

Jensen, K. B. (2008). Intermediality. In: Jensen, K. B., Craig, R. T. (Hrsg.): The International Encyclopedia of Communication Theory and Philosophy. Oxford: Wiley Blackwell. Zugriff: https://onlinelibrary.wiley.com (Abruf: 31.05.2019). 
Kinder, M. (1991). Playing with power in movies, television, and video games. Berkeley, Los Angeles, London: UC Press.

Kirschner, H. (2013). Go Live! Der User-Livestream als Präsentationsbühne. In: Lucht, P.; Schmidt, L. M.; Tuma, R. (Hrsg.): Visuelles Wissen und Bilder des Sozialen. Aktuelle Entwicklungen in der Soziologie des Visuellen. (S. 157-174). Wiesbaden: Springer.

Schneider, J. G. (2017). Medien als Verfahren der Zeichenprozessierung: Grundsätzliche Überlegungen zum Medienbegriff und ihre Relevanz für die Gesprächsforschung. Gesprächsforschung - Online-Zeitschrift zur verbalen Interaktion (18), 34-55.

Schneider, J. G./ Stöckl, H. (2011). Medientheorien und Multimodalität: Zur Einführung. In: Schneider, J. G.; Stöckl, H. (Hrsg.): Medientheorien und Multimodalität. (S. 10-38). Köln: Herbert von Halem.

Stöckl, H. (2004). Die Sprache im Bild - das Bild in der Sprache. Zur Verknüpfung von Sprache und Bild im massenmedialen Text. Konzepte - Theorien Analysemethoden. Berlin, New York: De Gruyter.

Storrer, A. (2008). Hypertextlinguistik. In: Janich, N. (Hrsg.): Textlinguistik: 15 Einführungen. (S. 315-332). Tübingen: Narr.

Wasilewska-Chmura, M. (2011). Przestrzeń intermedialna literatury i muzyki. Kraków: WUJ.

Wolf, W. (2002). Intermediality Revisited. Reflections on Word and Music Relations in the Context of a General Typology of Intermediality. In: Lodato, S. M.; Aspden, S., Bernhart, W. (Hrsg.): Word and Music Studies: Essays in Honor of Steven Paul Scher and on Cultural Identity and the Musical Stage. Word and Music Studies (4). (S. 13-34). Amsterdam: Rodopi.

Internetquellen:

1. https://www.duden.de/rechtschreibung/Livestream (Zugriff: 30.12.2019) 